\title{
Revisiting the Efficacy and Tolerability of Topiramate for Tic Disorders: A Meta-Analysis
}

\author{
Liping Yu, MS, Junjuan Yan, MS, Fang Wen, MS, Fang Wang, MS, \\ Jingran Liu, MD, Yonghua Cui, MD, ${ }^{*}$ and Ying Li, PhD*
}

\begin{abstract}
Background: Tic disorders (TDs) are chronic neuropsychiatric disorders that usually begin in childhood. Currently, antipsychotic drugs, such as aripiprazole and risperidone, are frequently used to treat TD. However, adverse reactions limit their further usage. The efficacy of topiramate has been reported in recent studies. This study aimed to investigate the efficacy and tolerability of topiramate in the treatment of children with TDs.

Methods: In this study, we performed a meta-analysis to assess the efficacy and tolerability of topiramate for TDs and identified the randomized controlled trials to evaluate topiramate for children with TDs from PubMed, the China National Knowledge Infrastructure database, Web of Science, and relevant reference lists. Quality assessment followed the Cochrane Handbook for Systematic Reviews of Interventions. A modified Jadad scale was used to assess the quality of included studies. Risk ratio (RR) was calculated as the effect size of efficacy and tolerability of topiramate for TDs.

Results: A total of 15 studies involving 1070 participants aged 2-17 were included in the present meta-analysis, and 693 (64.71\%) participants were male cases, with 14 studies evaluating the efficacy of topiramate for the treatment of TD. The results suggested that topiramate was more effective than control drugs (RR: $1.13,95 \%$ confidence interval: [1.06-1.20], $I^{2}=36 \%, Q=20.31$, $p=0.09$ ). For the adverse events, there were 15 trials included in the meta-analysis, and the results showed that topiramate had fewer adverse events than control drugs (RR: 0.54, 95\% confidence interval: [0.46-0.65], $I^{2}=45 \%, Q=25.49, p=0.03$ ).

Conclusion: Compared with haloperidol and tiapride, topiramate appears to be a promising medication with good efficacy and tolerability for children with TDs. In future studies, large-sample, double-blind, placebo-controlled trials are needed to confirm its efficacy and tolerability compared with atypical antipsychotic agents (such as risperidone or aripiprazole).
\end{abstract}

Keywords: topiramate, tic disorders, Tourette syndrome, children

\section{Introduction}

$\mathbf{T}^{\prime}$ IC DISORDERS (TDS) ARE a chronic complex neurodevelopmental movement disorder that is mainly characterized by multiple motor and phonic tics. Yang et al. (2016) found that the prevalence of tic disorders among children in China is $6.1 \%$. Antipsychotic drugs, such as risperidone, haloperidol, tiapride, and sulpiride, are widely used for treatment of TDs. However, their use is limited because of the frequent occurrence of adverse events, such as extrapyramidal reactions, overweight, and nausea (Quezada and Coffman 2018). Thus, there is an unmet need for more effective and safer drugs for TDs treatment, especially in children.

Topiramate, an adjunctive and monotherapy drug for seizures, is widely used due to its broad-spectrum antiepileptic effect (Nevitt et al. 2019). Topiramate may be associated with potentiating the activity of the inhibitory neurotransmitter $\gamma$-aminobutyric acid and blocking neuronal excitation mediated by glutamate, which could express $\alpha$-amino-3-hydroxy-5-methylisoxazole-4-propionic acid (AMPA) and kainic acid and its receptors (Okuyama et al. 2016). Compared with placebo, topiramate has shown a statistically significant reduction in Yale Global Tic Severity Scale (YGTSS) scores (Jankovic et al. 2010). Two meta-analyses confirmed that the efficacy of topiramate for TDs is better than that of haloperidol or tiapride and had fewer adverse events (Yang et al. 2013; Zheng et al. 2015). However, inconsistent results were also reported. For example, Su et al. (2015) reported that topiramate showed more side effects than a Chinese herb and aripiprazole. Moreover, there have been several new randomized controlled trials (RCTs) from China on topiramate for TDs in recent years. Therefore, it is important to further examine the efficacy and safety of topiramate.

In the present study, meta-analysis was used to assess the efficacy and safety of topiramate for TDs. The risk ratio (RR) was used as the effect size for the efficacy and safety.

Department of Psychiatry, Beijing Children's Hospital, Capital Medical University, National Center for Children's Health, Beijing, China. *These two authors contributed equally to this work.

Funding: The Special Fund of the Pediatric Medical Coordinated Development Center of Beijing Hospitals Authority, No. XTYB201802. 


\section{Materials and Methods}

\section{Search strategy}

We searched PubMed, the China National Knowledge Infrastructure, and Web of Science. We also screened the bibliographies of relevant articles to identify relevant studies. The terms "topiramate" or "topamax" and "Tourette syndrome" or "tic" were used to search for related studies. Two reviewers independently screened and crosschecked the literature based on the inclusion criteria. Two evaluators independently checked the extracted data from the included studies.

\section{Inclusion and exclusion criteria}

Inclusion criteria. (1) All the RCTs for the treatments of TDs were included when comparing topiramate with other medications. (2) Participants had to have a clinical diagnosis of TDs based on the Diagnostic and Statistical Manual of Mental Disorders, 4th ed. (DSM-IV; American Psychiatric Association 1994) or Diagnostic and Statistical Manual of Mental Disorders, 5th ed. (DSM-5; American Psychiatric Association 2013), the Chinese Classification and Diagnostic Criteria of Mental Disorders Version 3, or the International Classification of Diseases, Tenth Revision. (3) All participants were younger than 18 years of age.

Exclusion criteria. (1) The studies were not RCTs. (2) The outcome measurement was not YGTSS, or the number of successful cases and the number of patients with adverse reactions were not reported.

\section{Outcome measures used to quantify treatment effects}

We used the YGTSS score as the outcome measurement. The number of effective treatments was calculated by the evaluation of effective mitigation, mainly depending on clinical efficacy determination based on control of tics, including disappearance or relief of tics. The "effective treatment" should meet the criteria list below: the pretreatment YGTSS score minus the posttreatment score divided by the pretreatments YGTSS score was larger than $30 \%$. The Treatment Emergent Symptom Scale was used to assess the side effects. Any patients showed any side effects were included in the "side effects group." In addition, the Jadad scale is widely used to assess the quality of RCTs (Jadad et al. 1996), and we used a modified Jadad scale including eight items to assess the included studies (more details see the head of Table 3).

\section{Data extraction}

The following characteristics were extracted: author, age, sample size (the percent of male), drug dose of treatment group and control group, treatment period, outcome measurements, and diagnosis criteria.

\section{Statistical analyses}

$\mathrm{R}$ version 3.4.1 was used to perform the meta-analysis. We calculated the RRs with $95 \%$ confidence intervals to identify the effect size of efficacy and safety. A $Q$ test was used to assess the heterogeneity of each meta-analysis. The potential sources of the heterogeneity were explored by subgroup analysis. Additionally, we used a funnel plot to detect the publication bias.

\section{Results}

\section{The included studies}

A total of 15 studies including 1070 participants, were identified based on the inclusion and exclusion criteria (Fig. 1). We list the author, age, sample size (the percentage of males), drug dose of the treatment group and control group, and related characteristics in Tables 1 and 2 .

It should be noted that all included studies are from China. Although each study was an RCT, only two studies reported the

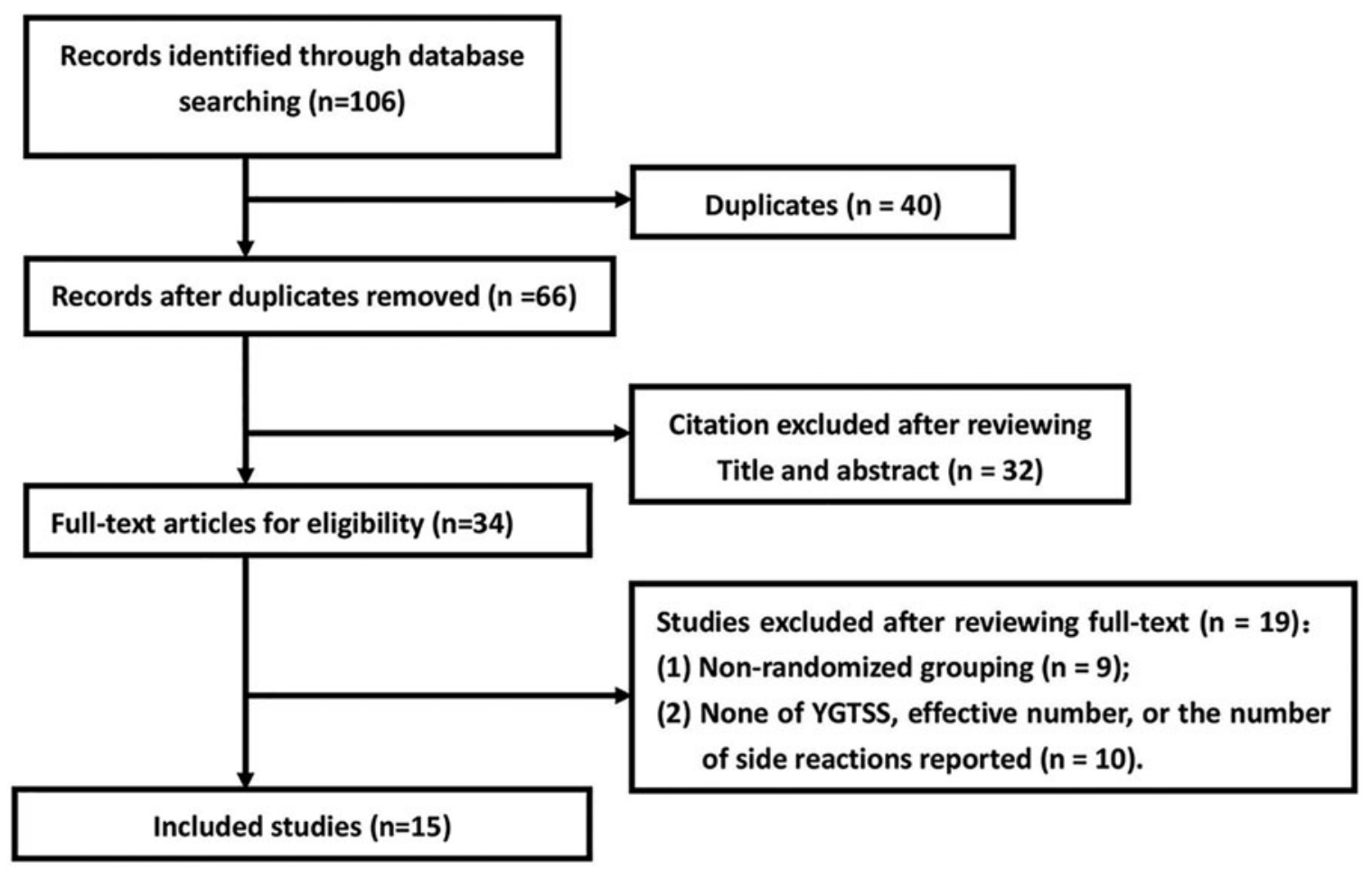

FIG. 1. The flow chart of the included study. 


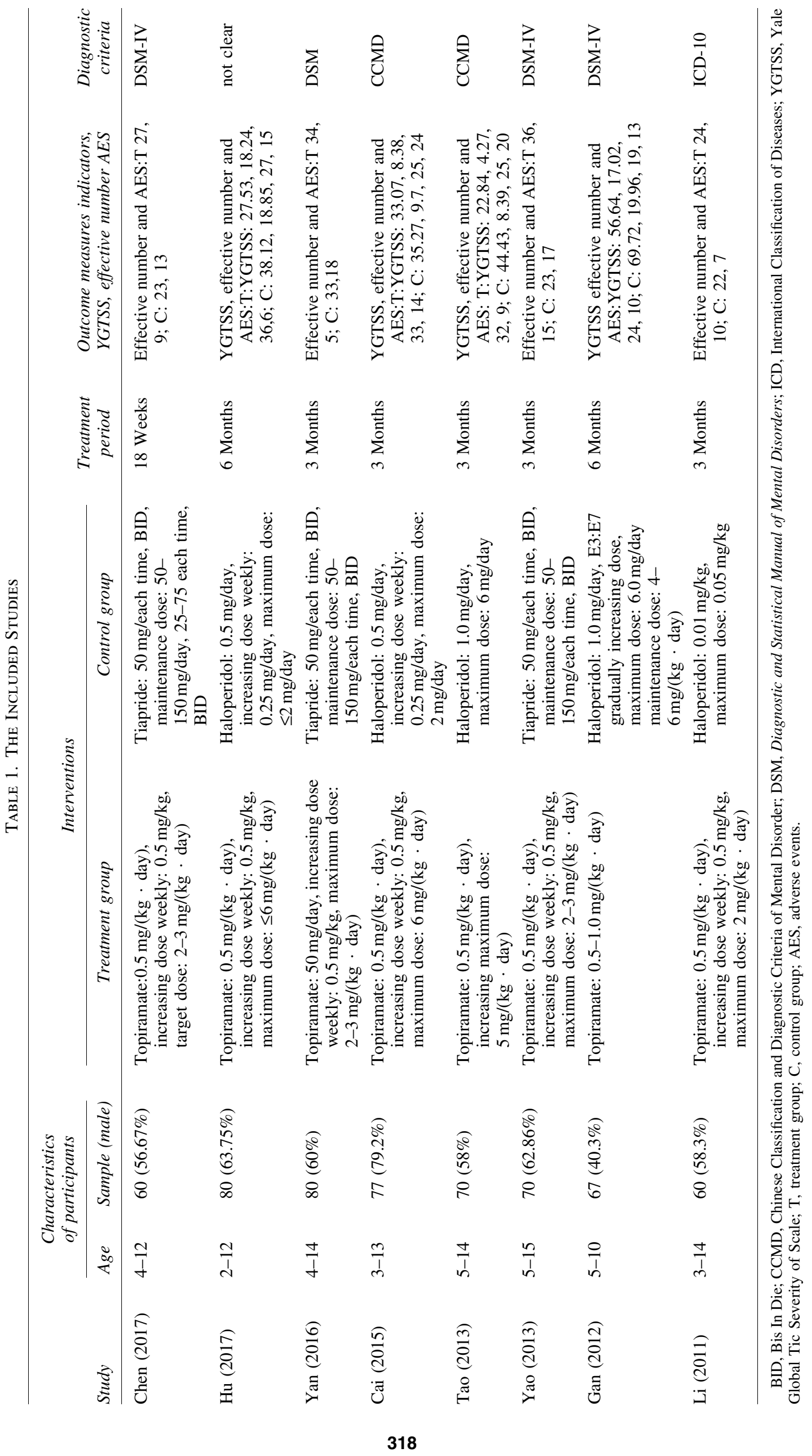




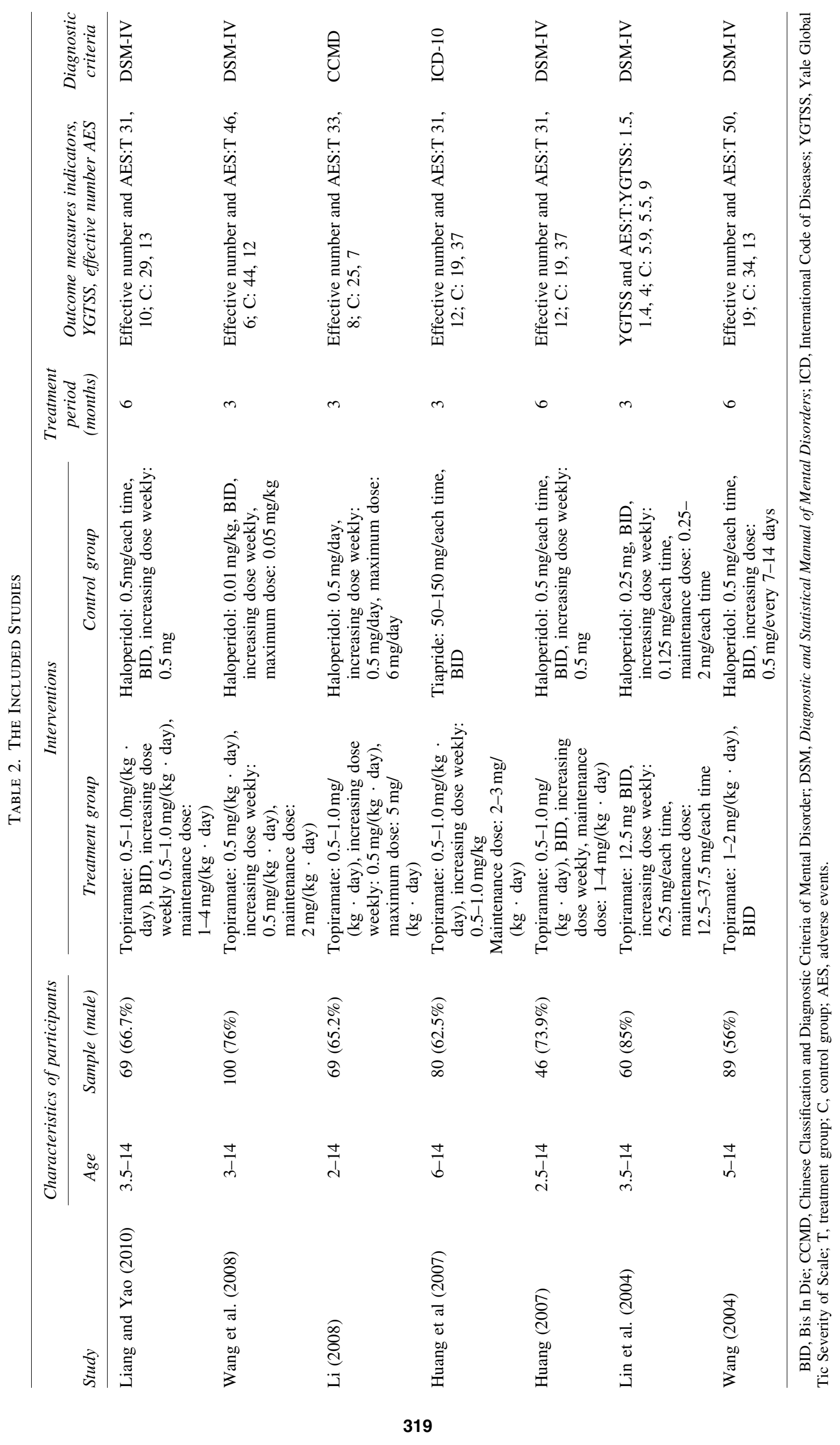




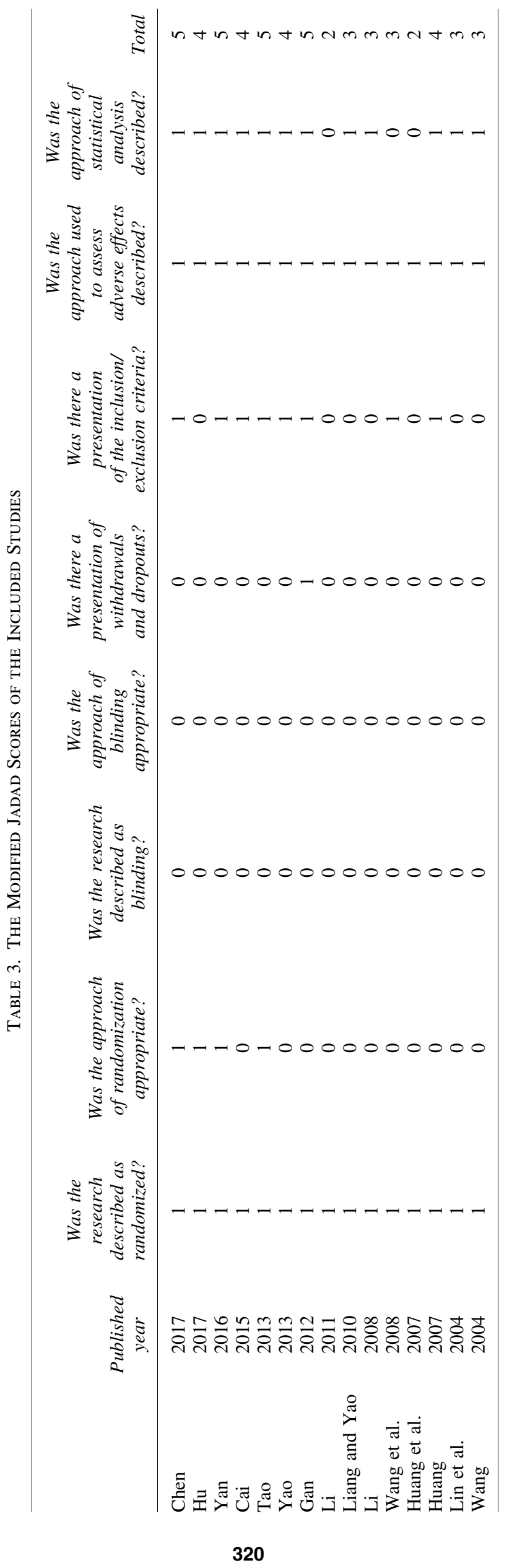




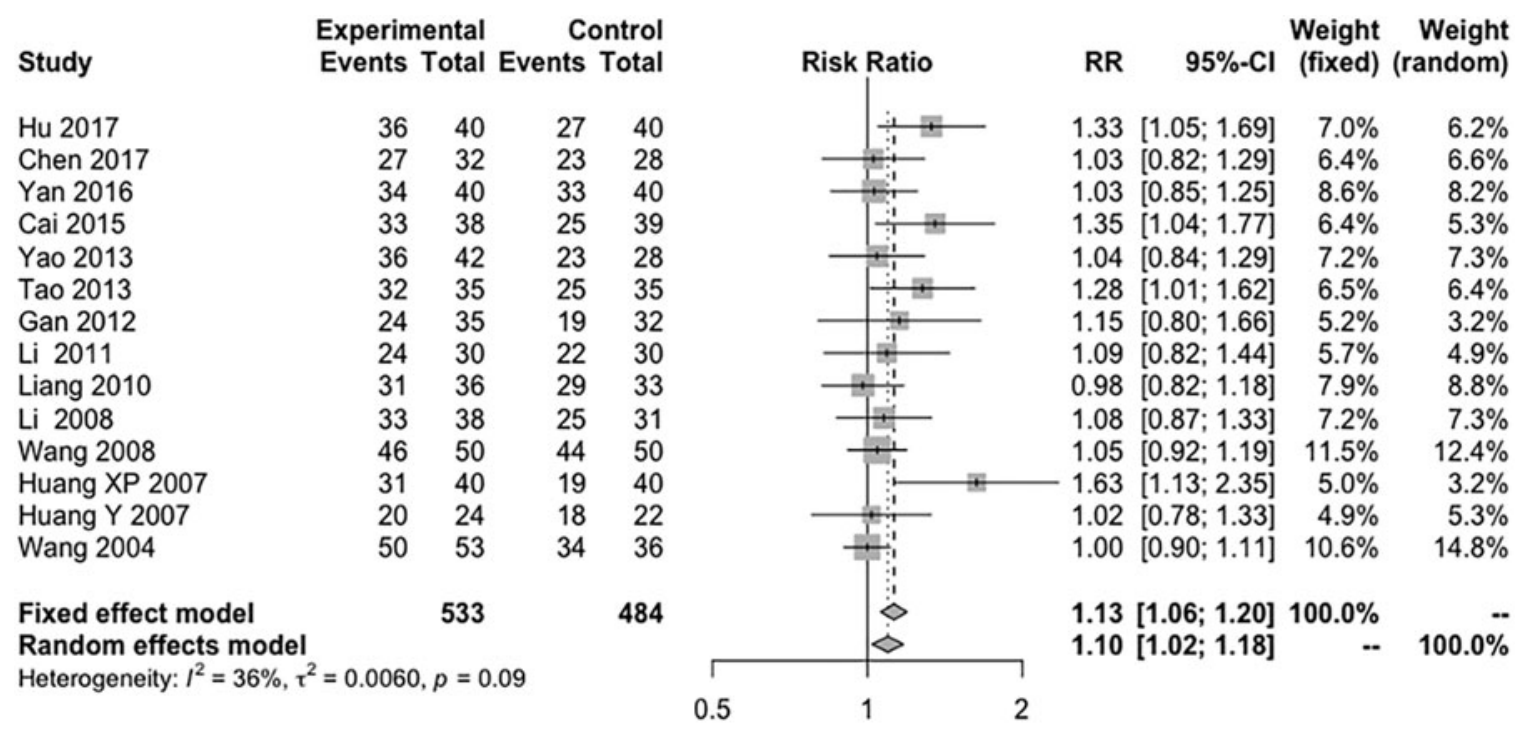

FIG. 2. Forest plots of the meta-analysis for the efficacy of topiramate.

random methods. The control drugs were haloperidol or tiapride. All studies were unclear about allocation concealment. Table 3 shows the quality assessments by a modified Jadad scale.

Additionally, from the Supplementary Figure S1 of funnel plot, we found that there might be no publication bias for the included studies.

\section{Meta-analysis of efficacy of topiramate for TDs}

We included 14 studies in the meta-analysis of efficacy. RR was calculated to compare the efficacy between topiramate and other control medications. The results showed that $\mathrm{RR}=1.13(95 \%$ CI: [1.06-1.20], $I^{2}=36 \%, Q=20.31, p=0.09$ ) based on fixed effect model, which indicated that the efficacy of topiramate was better than that of control medications. Although the heterogeneity of the results was not high, we still used subgroup analysis by different comparison conditions to explore the heterogeneity. The test for between-subgroup differences showed $Q=0.08$ ( $p=0.78)$ based on a fixed random model, which indicated that the efficacy of topiramate might not be influenced by different conditions. For more details see Figures 2 and 3.

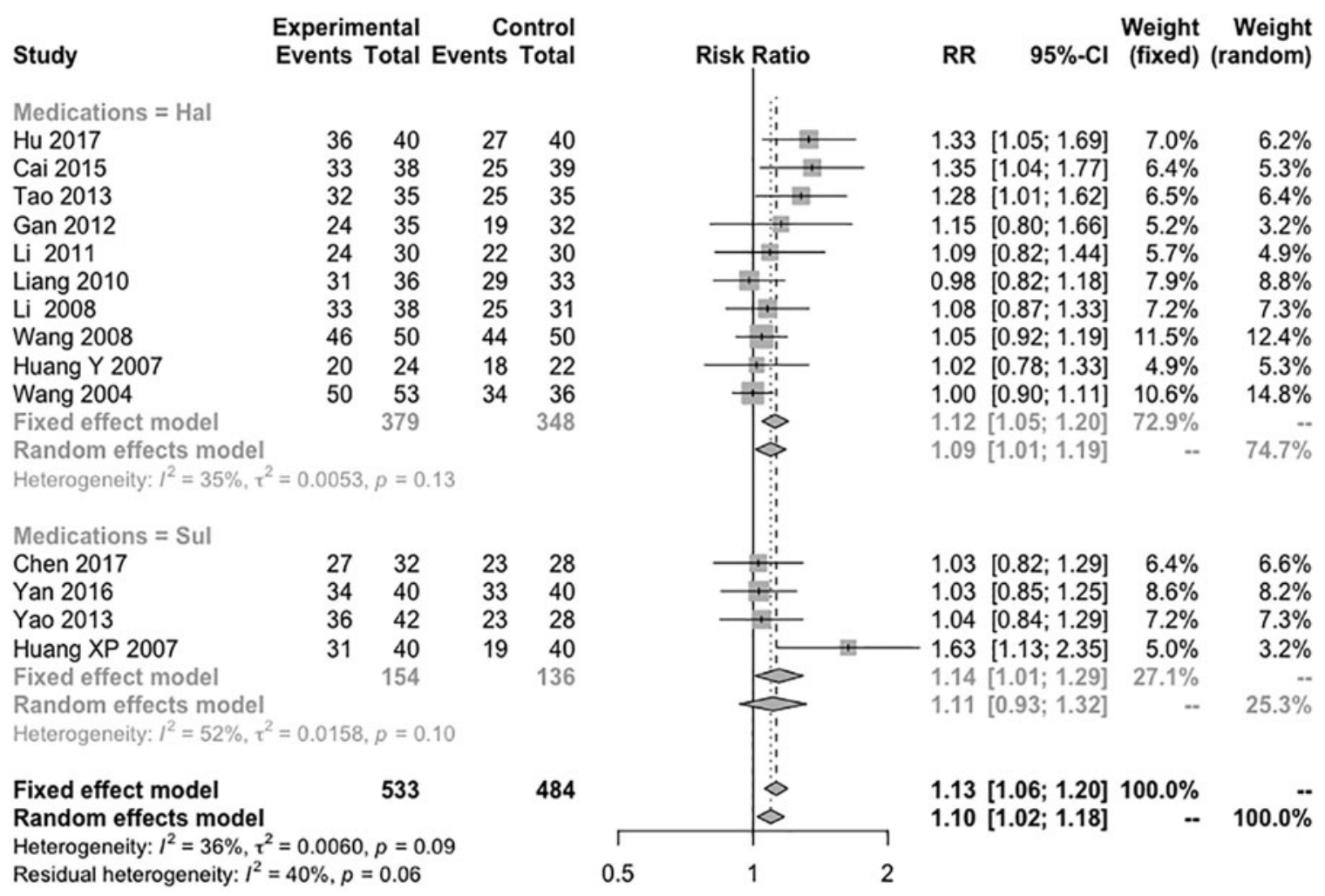

FIG. 3. Forest plots of subgroup analysis by comparison conditions for the efficacy of topiramate. 


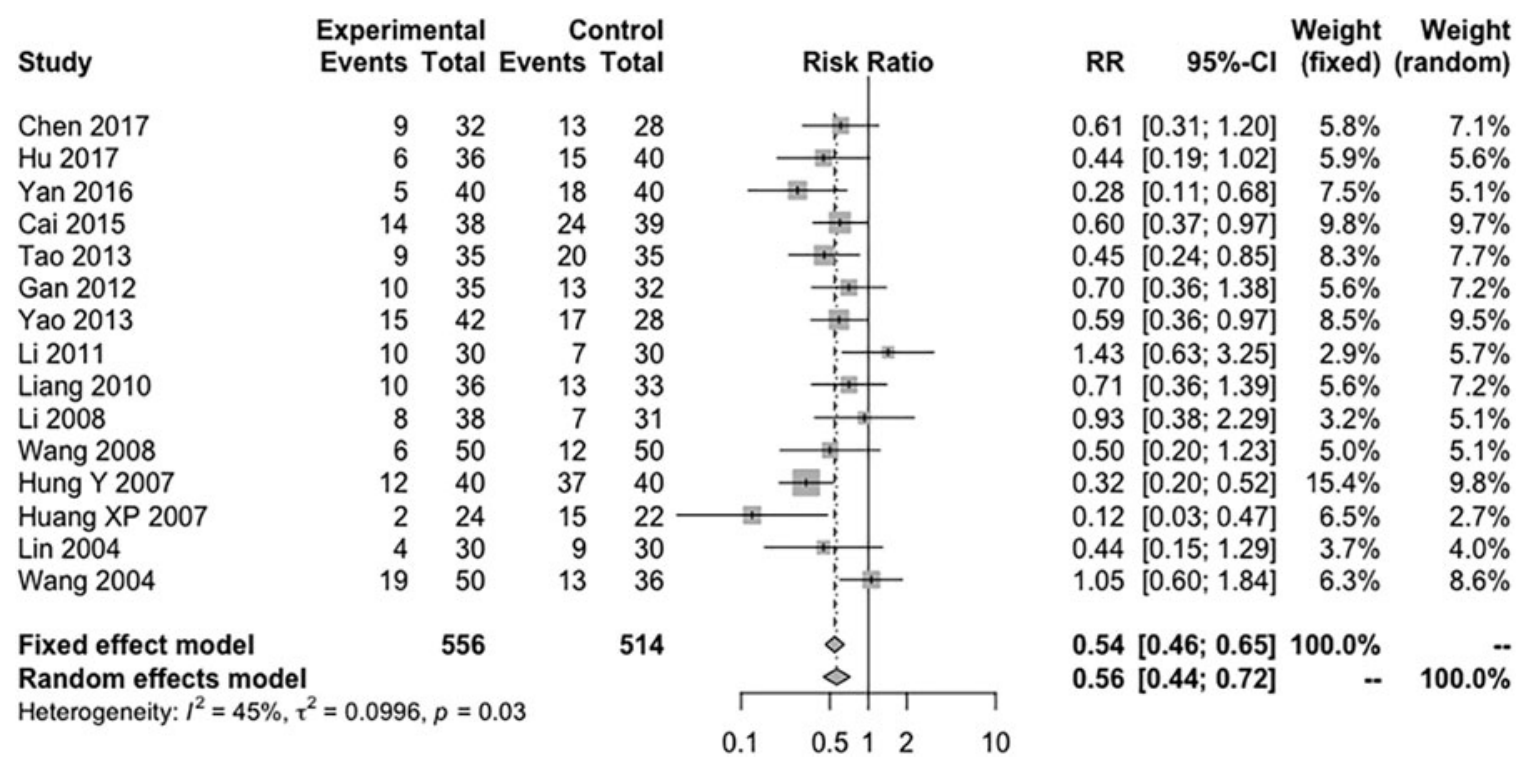

FIG. 4. Forest plots of meta-analysis of adverse events.

\section{Meta-analysis of safety topiramate for TDs}

We included all 15 studies in the meta-analysis of safety. The RR of adverse events was 0.56 , with $95 \%$ CI (0.44-0.72) based on a random effect model $\left(Q=25.49, I^{2}=45 \%\right.$, and $p=0.03$ ), which indicated that the heterogeneity was relatively high. Subgroup analysis by different comparison condition was used to identify the heterogeneity. The results showed that the residual heterogeneity was $38 \%(p=0.07)$. The test for subgroup difference showed that $Q=4.36(p=0.036)$. For the haloperidol subgroup, the RR was 0.62 , with $95 \%$ CI $(0.50$ $0.77)$ and $I^{2}=38 \%(p=0.09)$. For the tiapride subgroup, the RR was 0.42 , with $95 \%$ CI $(0.31-0.56)$ and $I^{2}=37 \%(p=0.19)$. These results indicated that different comparison conditions

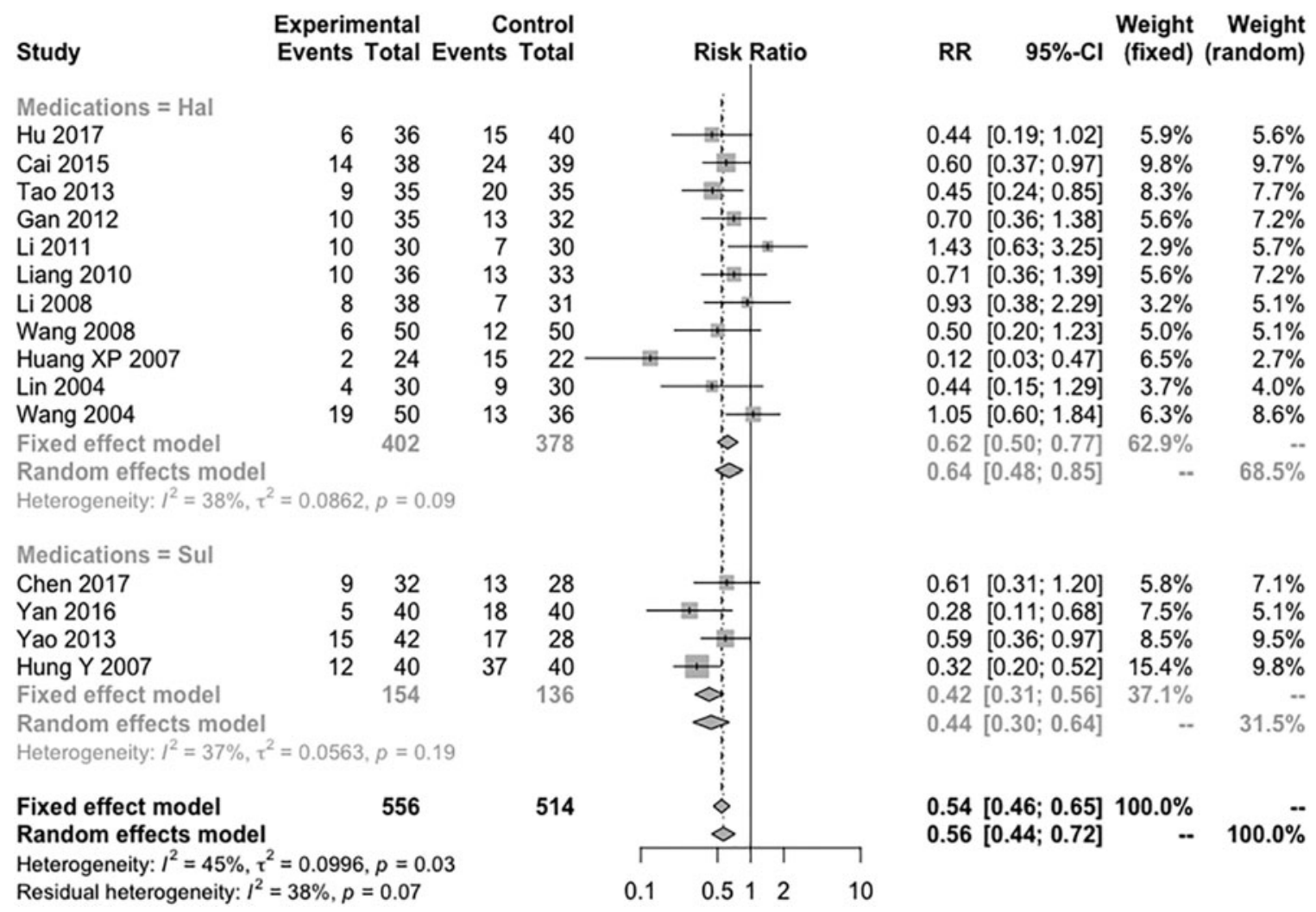

FIG. 5. Forest plots of subgroup analysis by comparison condition for the safety of topiramate. 
might influence the safety of topiramate. More details see Figures 4 and 5.

\section{Discussion}

This meta-analysis confirmed the efficacy of topiramate in the treatment of tics. Compared with haloperidol and tiapride, topiramate has better efficacy and fewer adverse effects in the treatment of tics. Our findings provide new evidence for the usage of topiramate by clinicians. Currently, atypical antipsychotics (such as aripiprazole) are widely used for TDs (Wang et al. 2017; Yang et al. 2019). For example, aripiprazole is considered the first-line medication for the management of tics in Japan (Hamamoto et al. 2019). To our knowledge, only one study with small sample has compared aripiprazole with topiramate, and no significant difference was identified between them (Wang 2015). Therefore, much more evidence is needed to confirm the efficacy of topiramate compared with aripiprazole or risperidone.

In the present study, we also demonstrated that topiramate is well-tolerated compared with tiapride and haloperidol. The adverse events of topiramate can include drowsiness or fatigue, headache, akathisia, gastrointestinal disturbance, and anxiety or depression (Nevitt et al. 2019). However, few cases have reported severe adverse effects of topiramate for TDs (Jankovic et al. 2010; Kuo and Jimenez-Shahed 2010). In contrast, extrapyramidal side effects and weight gain are reported consistently for antipsychotics and could lead to severe functional impairment for patients with TDs (Narula et al. 2010; Oliveira et al. 2019). In should be noted that, for some treatment-resistant cases, a high dosage of antipsychotics is always used, but weight gain will occur and can become a barrier for their further usage. Interestingly, some studies have reported that topiramate could enhance glucose transport and insulin action and suppress appetite through hypothalamic AMPA receptor antagonism (Okuyama et al. 2016; Cicek et al. 2018). Thus, topiramate can be used as an adjuvant with atypical antipsychotics in TDs patients who experience weight gain, especially for treatment-resistant ones, along with the augmentation of antipsychotics. Additionally, due to its clinical effect of reducing body weight, it is also promising for the management of TDs patients with obesity (Guerdjikova et al. 2018). However, other side effects such as anorexia, paresthesia, renal lithiasis, or mental clouding were also can be identified in some clinical cases (Nevitt et al. 2019). For its treatment for TD, we also need to pay more attentions to them.

It should be noted that the most common side effects for topiramate were language problems, cognitive disorder, kidney stones, and weight loss, while the rare side effects such as fatigure, insomnia, dizziness, diarrhea, and migraine also needed to be considered (Dell'Orto et al. 2014). In addition, the well-tolerated dose of topiramate was $25-150 \mathrm{mg} /$ day, but over $200 \mathrm{mg} / \mathrm{day}$, the side effects increased (Donegan et al. 2015; Pringsheim et al. 2019).

Moreover, as the glutamate modulators or anticonvulsants, topiramate could be considered as a potential drug for the treatment of obsessive-compulsive disorder (OCD) (Kariuki-Nyuthe et al. 2014; Marinova et al. 2017; Vlcek et al. 2018). Many studies indicated topiramate could be used for the OCD, especially for the Treatment-Resistant OCD (Van Ameringen et al. 2006; Mowla et al. 2010; Berlin et al. 2011; Afshar et al. 2014). Recently, a metaanalysis provided confirmed evidence that topiramate can be used as an augmentation drug for treatment-resistant compulsive disorders (Zhou et al. 2019). Obsessive-compulsive symptoms are frequently associated with TDs (Rothenberger and Roessner 2019).
The etiologies of OCD and TDs are thought to be shared and closely related (Perez-Vigil et al. 2016). This suggests that topiramate might be a potential choice for the treatment of TD with OCD in the future.

Notably, European Clinical Guidelines and Canadian guidelines recommended cognitive-behavioral treatment including habit reversal training (HRT) and exposure and response prevention as initial treatment for TD (van de Griendt et al. 2013). Recently, the American Academy of Neurology practice guideline updated new treatments for the TDs (Pringsheim et al. 2019). It also suggested that the behavioral therapy (such as HRT) and alpha agonists (including guanfacine and clonidine) were the first recommendations for the mild to moderate TDs. However, in this updated guideline, topiramate was suggested to use in patients with mild but troublesome tics who were not obtaining a satisfactory response or experienced adverse effects from other treatments, but physicians must take more attention for its common adverse effects.

Our study has two limitations. First, the quality of some included studies was poor; more high-quality RCTs are required. Second, the included studies were all from China, which reduces the generalizability of the findings.

\section{Conclusion}

In brief, topiramate is more effective than haloperidol and tiapride. Topiramate is particularly more promising in the treatment of TDs cases with obesity or OCD. However, there is a lack of sufficient evidence for the comparison of efficacy and safety between topiramate and atypical antipsychotics (such as risperidone or aripiprazole). In the future, we need larger samples and more high-quality studies to confirm these findings.

\section{Clinical Significance}

Topiramate is promising in the treatment of TDs. It showed better efficacy and safety when compared with haloperidol and tiapride.

\section{Disclosures}

No competing financial interests exist.

\section{Supplementary Material}

\author{
Supplementary Figure S1
}

\section{References}

Afshar H, Akuchekian S, Mahaky B, Zarean E: Topiramate augmentation in refractory obsessive-compulsive disorder: A randomized, double-blind, placebo-controlled trial. J Res Med Sci 19: 976-981, 2014.

American Psychiatric Association: Diagnostic and Statistical Manual of Mental Disorders, 4th ed. Washington, DC: American Psychiatric Association; 1994.

American Psychiatric Association. Diagnostic and Statistical Manual of Mental Disorders, 5th ed. Washington, DC: American Psychiatric Association; 2013.

Berlin HA, Koran LM, Jenike MA, Shapira NA, Hollander E: Double-blind, placebo-controlled trial of topiramate augmentation in treatment-resistant obsessive-compulsive disorder. J Clin Psychiatry 72:716-721, 2011. 
Cai X: Effect of topiramate on children with multiple tic disorder. J Pract Med 31:1702-1704, 2015 (Article in Chinese).

Chen J: Comparison of clinical efficacy and safety between topiramate and thiophene in the treatment of multiple tic disorder in children. J Pediatr Pharm 23:20-22, 2017 (Article in Chinese).

Cicek NP, Kamasak T, Serin M, Okten A, Alver A, Cansu A: The effects of valproate and topiramate use on serum insulin, leptin, neuropeptide $\mathrm{Y}$ and ghrelin levels in epileptic children. Seizure 58: 90-95, 2018.

Dell'Orto VG, Belotti EA, Goeggel-Simonetti B, Simonetti GD, Ramelli GP, Bianchetti MG, Lava SA: Metabolic disturbances and renal stone promotion on treatment with topiramate: A systematic review. Br J Clin Pharmacol 77:958-964, 2014.

Donegan S, Dixon P, Hemming K, Tudur-Smith C, Marson A: A systematic review of placebo-controlled trials of topiramate: How useful is a multiple-indications review for evaluating the adverse events of an antiepileptic drug? Epilepsia 56:1910 1920, 2015.

Gan R: Clinical observation on the role of topiramate in the treatment of child Tourette's syndrome. Hei Long Jiang Med 36:458-460, 2012 (Article in Chinese).

Guerdjikova AI, Williams S, Blom TJ, Mori N, McElroy SL: Combination phentermine-topiramate extended release for the treatment of binge eating disorder: An open-label, prospective study. Innov Clin Neurosci 15:17-21, 2018.

Hamamoto Y, Fujio M, Nonaka M, Matsuda N, Kono T, Kano Y: Expert consensus on pharmacotherapy for tic disorders in Japan. Brain Dev 41:501-506, 2019.

$\mathrm{Hu} \mathrm{H}$ : Clinical analysis of topiramate in the treatment of multiple tic disorder in children. J Shanxi Med Coll Continuing Educ 27:37-39, 2017 (Article in Chinese).

Huang X: The clinic efficacy of topamax in the treatment of Tourette's syndrome. Med J West China 19:261-262, 2007 (Article in Chinese).

Huang Y, Wang J, Fang P: Clinical study of topiramate in treating with childhood Tourette's syndrome. North SiChuan Med Coll 22: 437-439, 2007 (Article in Chinese).

Jadad AR, Moore RA, Carroll D, Jenkinson C, Reynolds DJ, Gavaghan DJ, McQuay HJ: Assessing the quality of reports of randomized clinical trials: Is blinding necessary? Control Clin Trials 17:1-12, 1996.

Jankovic J, Jimenez-Shahed J, Brown L: A randomised, double-blind, placebo-controlled study of topiramate in the treatment of Tourette syndrome. Neuro Neurosurq Psychiatry 81:70-73, 2010.

Kariuki-Nyuthe C, Gomez-Mancilla B, Stein DJ: Obsessive compulsive disorder and the glutamatergic system. Curr Opin Psychiatry 27:32-37, 2014.

Kuo S, Jimenez-Shahed J: Topiramate in treatment of tourette syndrome. Clin Neuropharmacol 33:32-34, 2010.

Li A: The efficacy analysis of topiramate in treatment of Tourette's syndrome in children [in Chinese]. China Foreign Med 1:129, 2011.

Li J: The efficacy evaluation of topiramate in treating with childhood Tourette's syndrome. Strait Pharmaceut 20:114-115, 2008 (Article in Chinese).

Liang G, Yao G: The efficacy of Topamax in the treatment of Tourette's syndrome. Pract Med 26:4192-4193, 2010 (Article in Chinese).

Lin Y, Lin G, Lin J, Wu Y, Qiu Y: The comparison study of the therapeutic effect of topiramate and haloperidol in children with Tourette syndrome. Xinxiang Med Coll 21:351-353, 2004 (Article in Chinese).

Marinova Z, Chuang DM, Fineberg N: Glutamate-modulating drugs as a potential therapeutic strategy in obsessive-compulsive disorder. Curr Neuropharmacol 15:977-995, 2017.
Mowla A, Khajeian AM, Sahraian A, Chohedri AH, Kashkoli F: Topiramate augmentation in resistant OCD: A double-blind placebocontrolled clinical trial. CNS Spectr 15:613-617, 2010.

Narula PK, Rehan HS, Unni KE, Gupta N: Topiramate for prevention of olanzapine associated weight gain and metabolic dysfunction in schizophrenia: A double-blind, placebo-controlled trial. Schizophr Res 118:218-223, 2010.

Nevitt SJ, Sudell M, Tudur Smith C, Marson AG: Topiramate versus carbamazepine monotherapy for epilepsy: An individual participant data review. Cochrane Database Syst Rev CD012065, 2019.

Okuyama Y, Oya K, Matsunaga S, Kishi T, Iwata N: Efficacy and tolerability of topiramate-augmentation therapy for schizophrenia: A systematic review and meta-analysis of randomized controlled trials. Neuropsychiatr Dis Treat 12:3221-3236, 2016.

Oliveira B, Ferrari P, Herrerias B, Hirai F, Gracitelli C: The use of topiramate for weight loss causing acute glaucoma: A case report and literature review. Med Hypothesis Discov Innov Ophthalmol 8: 116-120, 2019.

Perez-Vigil A, Fernandez de la Cruz L, Brander G, Isomura K, Gromark C, Mataix-Cols D: The link between autoimmune diseases and obsessive-compulsive and tic disorders: A systematic review. Neurosci Biobehav Rev 71:542-562, 2016.

Pringsheim T, Okun MS, Müller-Vahl K, Martino D, Jankovic J, Cavanna AE, Woods DW, Robinson M, Jarvie E, Roessner V: Practice guideline recommendations summary: Treatment of tics in people with Tourette syndrome and chronic tic disorders. Neurology 92:896-906, 2019.

Quezada J, Coffman KA: Current approaches and new developments in the pharmacological management of Tourette syndrome. CNS Drugs 32:33-45, 2018.

Rothenberger A, Roessner V: Psychopharmacotherapy of obsessivecompulsive symptoms within the framework of Tourette syndrome. Curr Neuropharmacol 17:703-709, 2019.

Su H: A controlled study of the clinical effects of duodongning capsule,topiramate and aripiprazole in the treatmen of Tic disorders. MSc Dissertation, 2015 (in Chinese).

Tao R: Effect of topiramate on children with tic disorder and plasma glutamate and aspartate levels in children. J Pediatr Pharm 19: 21-23, 2013 (Article in Chinese).

Van Ameringen M, Mancini C, Patterson B, Bennett M: Topiramate augmentation in treatment-resistant obsessive-compulsive disorder: A retrospective, open-label case series. Depress Anxiety 23: $1-5,2006$.

van de Griendt JMTM, Verdellen CWJ, Van Dijk MK, Verbraak MJPM: Behavioural treatment of tics: Habit reversal and exposure with response prevention. Neurosci Biobehav Rev 37:1172-1177, 2013.

Vlcek P, Polak J, Brunovsky M, Horacek J: Role of glutamatergic system in obsessive-compulsive disorder with possible therapeutic implications. Pharmacopsychiatry 51:229-242, 2018.

Wang G, Li M, Chen B: Clinical research of topiramate in treating with childhood Tourette's syndrome. Tianjin Pharm 20:52-53, 2008 (Article in Chinese).

Wang L: Therapeutic effect of aripiprazole and topiramate on refractory tic disorder. J Med Forum 36:139-140, 2015 (Article in Chinese).

Wang S, Wei YZ, Yang JH, Zhou YM, Cheng YH, Yang C, Zheng Y: The efficacy and safety of aripiprazole for tic disorders in children and adolescents: A systematic review and meta-analysis. Psychiatry Res 254:24-32, 2017.

Wang X: Clinical application of topiramate in children with multiple tic disorder. China Children's Health J 12:414, 422, 2004 (Article in Chinese). 
Yan J: Effect of topiramate on children with multiple tic disorder. Contemp Chinese Med 23:66-68, 2016 (Article in Chinese).

Yang C, Yi Q, Zhang L, Cui H, Mao J: Safety of aripiprazole for tics in children and adolescents: A systematic review and meta-analysis. Medicine 98:e15816, 2019.

Yang C, Zhang L, Zhu P, Zhu C, Guo Q: The prevalence of tic disorders for children in China: A systematic review and metaanalysis. Medicine 95:e4354, 2016.

Yang CS, Zhang LL, Zeng LN, Huang L, Liu YT: Topiramate for Tourette's syndrome in children: A meta-analysis. Pediatr Neurol 49:344-350, 2013.

Yao Y: Comparative analysis of topiramate and sulpiride in the treatment of multiple tic disorder in children. Guide China Med 11: 16-17, 2013 (Article in Chinese).

Zheng W, Zhang N, Yang J, Li X, Ma X, Xiang Y: Meta-analysis of topiramate and haloperidol in the treatment of children with tic disorder. Chinese J Child Health Care 23:303-306, 2015 (Article in Chinese).

Zhou DD, Zhou XX, Li Y, Zhang KF, Lv Z, Chen XR, Wan LY, Wang W, Wang GM, Li DQ, Ai M, Kuang L: Augmentation agents to serotonin reuptake inhibitors for treatment-resistant obsessivecompulsive disorder: A network meta-analysis. Prog Neuropsychopharmacol Biol Psychiatry 90:277-287, 2019.
Address correspondence to: Yonghua Cui, $M D$

Department of Psychiatry National Center for Children's Health Beijing Children's Hospital Capital Medical University

Nanlishi Road 56 Beijing 100045

China

E-mail: cuiyonghuapsy@126.com

Ying $\mathrm{Li}, \mathrm{PhD}$ Department of Psychiatry

National Center for Children's Health Beijing Children's Hospital Capital Medical University Nanlishi Road 56 Beijing 100045

China

E-mail: yyjnly@126.com 